\title{
Knight's Tours on Rectangular Chessboards Using External Squares
}

\author{
Grady Bullington, ${ }^{1}$ Linda Eroh, ${ }^{1}$ Steven J. Winters, ${ }^{1}$ and Garry L. Johns ${ }^{2}$ \\ ${ }^{1}$ University of Wisconsin Oshkosh, Oshkosh, WI 54901, USA \\ ${ }^{2}$ Saginaw Valley State University, University Center, MI 48710, USA \\ Correspondence should be addressed to Garry L. Johns; glj@svsu.edu
}

Received 27 May 2014; Revised 12 November 2014; Accepted 17 November 2014; Published 9 December 2014

Academic Editor: Stavros D. Nikolopoulos

Copyright (c) 2014 Grady Bullington et al. This is an open access article distributed under the Creative Commons Attribution License, which permits unrestricted use, distribution, and reproduction in any medium, provided the original work is properly cited.

\begin{abstract}
The classic puzzle of finding a closed knight's tour on a chessboard consists of moving a knight from square to square in such a way that it lands on every square once and returns to its starting point. The $8 \times 8$ chessboard can easily be extended to rectangular boards, and in 1991, A. Schwenk characterized all rectangular boards that have a closed knight's tour. More recently, Demaio and Hippchen investigated the impossible boards and determined the fewest number of squares that must be removed from a rectangular board so that the remaining board has a closed knight's tour. In this paper we define an extended closed knight's tour for a rectangular chessboard as a closed knight's tour that includes all squares of the board and possibly additional squares beyond the boundaries of the board and answer the following question: how many squares must be added to a rectangular chessboard so that the new board has a closed knight's tour?
\end{abstract}

\section{Introduction}

For many years individuals have been intrigued with the chessboard, not just because of the intricacies and strategies in the game of chess, but also for the many puzzles that have been devised on the board. In addition, mathematicians see these puzzles as special cases of major topics in graph theory. For instance, positioning queens on the board so that none can be captured illustrates independence; choosing the fewest number of squares so that rooks on these squares can attack every square is dominance; and moving a knight from square to square in such a way that it lands on every square once and returns to its starting point is an example of a Hamiltonian cycle. One source that surveys the history and results of many chessboard puzzles, as well as summarizing variations and extensions to other surfaces, is [1]. Specifically for the knight's tour problem, if we label each square on an $8 \times 8$ chessboard with an ordered pair using its row and column position, and assign $(1,1)$ to the upper left square, then a knight beginning at $(r, c)$ can legally move to any of the eight squares $(r \pm 2$, $c \pm 1)$ or $(r \pm 1, c \pm 2)$ unless $(r, c)$ is too close to the edge of the board. An open knight's tour (sometimes called a path) consists of a sequence of legal moves so that a knight lands on each square exactly once. See Figure 1 for some examples that will be used later where the tours begin at the square labeled $a$ or 1 and end at squares $l$ or 20. A closed knight's tour has the added condition that the knight must end its tour on the initial square. The $8 \times 8$ chessboard can easily be extended to rectangular boards, and in 1991, Schwenk characterized all rectangular boards that have a closed knight's tour [2]. He used an induction proof with nine basis steps to construct closed knight's tours for all admissible boards to prove the following.

Schwenk's Theorem. An $m \times n$ chessboard with $m \leq n$ has a closed knight's tour in all but the following cases:
(a) $m=1$ for all $n$;
(b) $m=2$ for all $n$;
(c) $m=3$ and $n=4,6,8$ or is odd;
(d) $m=4$ for all $n$;
(e) $m>4$ for all combinations of $m$ odd and $n$ odd. 


\begin{tabular}{|c|c|c|c|}
\hline$a$ & $d$ & $g$ & $j$ \\
\hline$l$ & $i$ & $b$ & $e$ \\
\hline$c$ & $f$ & $k$ & $h$ \\
\hline
\end{tabular}

\begin{tabular}{|c|c|c|c|c|}
\hline 20 & 1 & 16 & 7 & 12 \\
\hline 15 & 6 & 11 & 2 & 17 \\
\hline 10 & 19 & 4 & 13 & 8 \\
\hline 5 & 14 & 9 & 18 & 3 \\
\hline
\end{tabular}

FIGURE 1: The $3 \times 4$ and $4 \times 5$ rectangular boards with open knight's tours.

More recently, Demaio and Hippchen investigated the impossible boards in [3] and determined the fewest number of squares that must be removed from a rectangular board so that the remaining board has a closed knight's tour. If we define an extended closed knight's tour for a rectangular chessboard as a closed knight's tour that includes all squares of the board and possibly additional squares beyond the boundaries of the board, then our interest is to answer the following: how many squares must be added to a rectangular chessboard so that the new board has a closed knight's tour? For an $m \times n$ board, the minimum number, called the extended tour number, is denoted as $\tau(m, n)$. Every rectangular board has an extended knight's tour and the extended tour numbers are summarized in our main result.

Theorem 1. Every $m \times n$ chessboard with $0<m \leq n$ has an extended tour number of 0 except for the following:

(a) $\tau(1, n)=n+2$ for all $n$;

(b) $\tau(2, n)=6$ if $n$ is even and $n \neq 4$; otherwise, $\tau(2, n)=$ 4 ;

(c) $\tau(3,3)=\tau(3,5)=3 ; \tau(3,4)=\tau(3,6)=\tau(3,8)=2$; and $\tau(3, n)=1$ if $n \geq 7$ and is odd;

(d) $\tau(4, n)=2$ for all $n$;

(e) $\tau(m, n)=1$ if $m>4$ with $m$ and $n$ both being odd.

Within the last twenty-five years, much of the research on knight's tours have taken on the tone set by Schwenk [2] by being concerned about which boards allow knight's tours. Knuth's work in [4] on leapers (i.e., generalized knights) and Watkins' book [1] have played no small part in the subject's popularity either. A survey of the literature shows that knight's tours have been studied on a variety of shapes and dimensions (cf. [5-7]). Generalized knight's moves have been studied in [8-10]. In [1], extensions of Schwenk's Theorem were given for four alternative surfaces: the torus, cylinder, Möbius strip, and the Klein bottle. Recently, the authors in [11] considered the same four surfaces and determined the fewest number of squares that must be removed or added to insure a knight's tour.

\section{Proof of Main Result}

We will assume that the board is $m \times n$ with $0<m \leq n$, the upper left corner of each board is black, and the numbering for the extended knight's tour begins at that square. Since every closed knight's tour alternates between black and white squares, we have three useful observations about the number of additional squares needed.

(a) The total number of squares (original and additional) must be even and must consist of an equal number of black and white squares.

(b) An odd number of squares are needed to connect two squares of the same color.

(c) An even number of squares are needed to connect two squares of opposite color.

In $[2,3]$, where tours are restricted to squares on the original board, no closed knight's tour exists when $m=1$ or 2; however, by allowing additional squares, the extended tour numbers do exist and are generally larger than those for larger boards. In addition, the arrangement of the added squares is not unique. For instance, consider the three variations for the $2 \times$ 2 board shown in Figure 2. Probably the second configuration is preferred over the first because the additional squares are all adjacent to the original board, but the third arrangement will be used in case $(b)$ of the proof to show that $\tau(2,2)=6$ because of its simplicity, symmetry, and the ability to extend it to longer boards.

The proof will be organized by considering the cases (a)(e) from Schwenk's Theorem in order.

(a) The Case $m=1$. A special situation applies for the 1 $\times 1$ chessboard. One solution is to move to an additional square and simply return to the original square; however, if backtracking is to be avoided, at least three additional squares are needed (by observation (b)). One configuration that uses exactly three additional squares is in Figure 3 with the original $1 \times 1$ chessboard on square 1 .

For $m=1$ and $n \geq 2$, a useful variation of the rectangular board is a graph where each square is represented by a vertex (colored white or black) and an edge joins two vertices if a knight can move between the corresponding squares in a single move. Thus, if $m=1$ and $n \geq 2$, the $1 \times n$ board can be thought of as a graph with $n$ isolated vertices. A cycle is formed by selecting a vertex one at a time, either adding an odd number of new vertices after it if the next selected vertex has the same color, or adding an even number of vertices after it if the next selected vertex has the opposite color and repeating this between the last selected vertex and the first one. Note that at least twice on the cycle the vertex color will change, so $\tau(1, n) \geq(n-2)+2+2=n+2$ for $n \geq 2$. This bound would be achieved if a shortest cycle could be formed by selecting the black vertices in succession and inserting one vertex between each pair, inserting two vertices between the last black vertex and the first white vertex selected, inserting one vertex after each white vertex until all vertices are selected, and then adding two vertices between the last white vertex and the first black vertex. Since there are $w$ white squares and $b$ black squares, then $n=w+b$ and this shortest cycle includes $(w-1)+2+(b-1)+2=n+2$ new vertices. This is accomplished by the following process. 

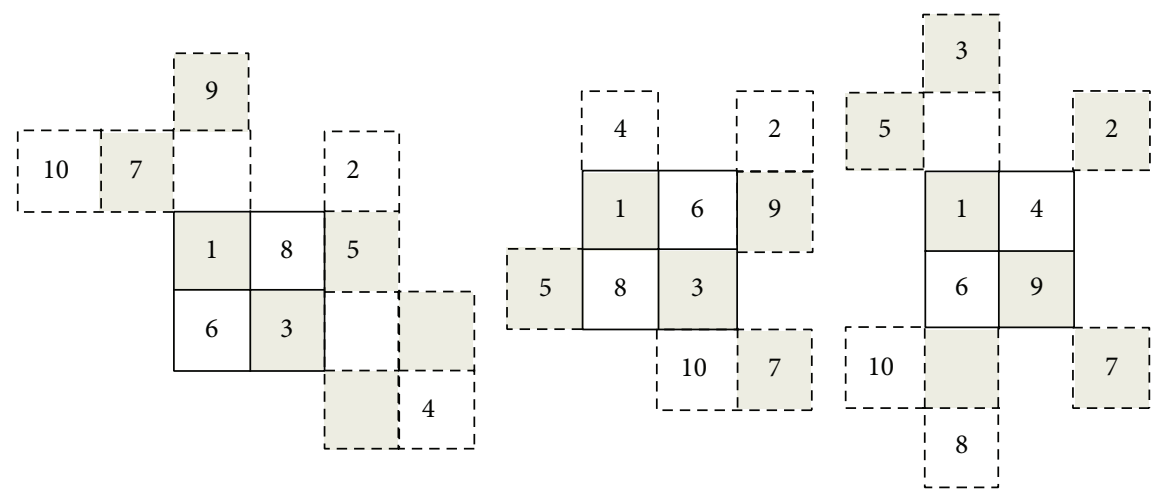

FIgURE 2: Three arrangements for the six additional vertices needed for the $2 \times 2$ board.

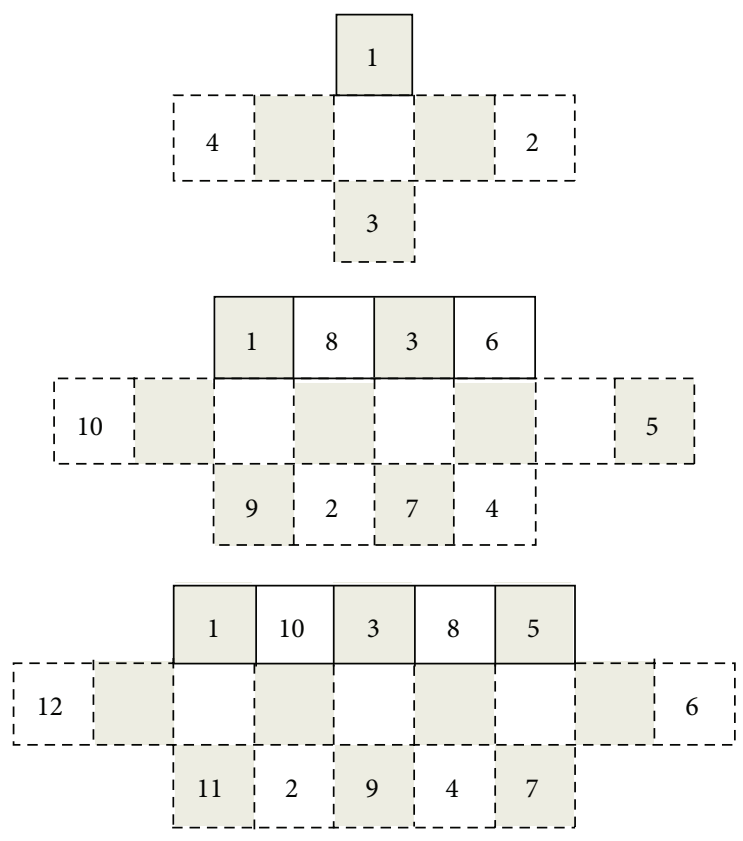

Figure 3: The $1 \times 1,1 \times 4$, and $1 \times 5$ rectangles.

(1) Place the $1 \times n$ board in the top row of a $3 \times(n+4)$ rectangle so that the upper left square of the board is in the $(1,3)$ position, and color that square black.

(2) Begin moving $(1,3),(3,4),(1,5), \ldots,(1, i),(3, i+$ $1),(1, i+2), \ldots,(1, m)$ where $m=n+1$ if $n$ is even or $n+2$ if $n$ is odd.

(3) If $n$ is even, continue with $(1, n+1),(3, n+2),(2, n+$ 4), $(1, n+2)$.

If $n$ is odd, continue with $(1, n+2),(2, n+4),(3, n+$ $2)$, and $(1, n+1)$.

(4) Now, proceed backwards from $(1, j),(3, j-1),(1, j-$ $2), \ldots,(1,4),(3,3),(2,1),(1,3)$ where $j=n+1$ if $n$ is odd or $n+2$ if $n$ is even.

The forward and backward passes through row 3 do not overlap because the first pass uses white squares and the reverse pass uses only black squares. (These cycles are illustrated in Figure 3 for $n=4$ and 5.) So, $\tau(1, n)=n+2$ for $n \geq 1$.

(b) The Case $m=2$. Consider that $n$ is even first. If $n=2$, then the corresponding graph consists of four isolated vertices, and if $n>4$ is even, then the corresponding graph consists of four paths with at least four additional vertices needed to connect them. If $n=2+4 k$ for some nonnegative integer $k$, then the paths are from $(1,1)$ to $(1, n-1),(2,1)$ to $(2, n-1),(1,2)$ to $(1$, $n)$, and $(2,2)$ to $(2, n)$. Notice that two of the paths begin (on the left) and end (on the right) on black squares, while two of the paths begin and end on white squares. On the other hand, if $n=4 k$ for some positive integer $k \geq 2$, then the paths are from $(1,1)$ to $(2, n-1),(2,1)$ to $(1, n-1),(1,2)$ to $(2, n)$, and $(2,2)$ to $(1, n)$. In this case, all four of the paths begin (on the left) and end (on the right) on squares of opposite colors. Let $P_{(1,1)}, P_{(1,2)}, P_{(2,1)}$, and $P_{(2,2)}$ denote the four paths where the subscript indicates the beginning vertex.

For each of these values of $n$ it is possible to link the four paths (or isolated vertices) together with exactly four additional vertices; however, then $P_{(1,1)}$ and $P_{(2,2)}$ will be joined at both ends and $P_{(1,2)}$ and $P_{(2,1)}$ will be joined at both ends, creating two disjoint cycles instead of a single knight's tour. Therefore, to create a single cycle, we will have to connect a white square to a white square, a black square to a black square, a white square to a black square, and a black square to a white square requiring at least 6 additional squares by observations (b) and (c). Notice also that for $n$ at least 6 , joining the beginning of one path to the end of another would require at least 2 additional squares, so we assume that we join the beginning of one path to the beginning of another (or the right end of one path to the right end of another). This can be done as follows: Connect $(1,1)$ to $(2,2)$ using the additional square $(3,0)$, connect $(1,2)$ to $(2,1)$ using the additional square $(0,0)$, connect $(1, n-1)$ to $(1, n)$ using $(0, n$ $+1)$ and $(-1, n-1)$, and connect $(2, n-1)$ to $(2, n)$ using $(3, n$ $+1)$ and $(4, n-1)$. Thus, only the six additional squares $(3,0)$, $(0,0),(0, n+1),(-1, n-1),(3, n+1)$, and $(4, n-1)$ are needed. For example, for the $2 \times 2$ board, the six external squares are $(3,0),(0,0),(0,3),(-1,1),(3,3)$, and $(4,1)$ and a tour is $(1,1)$ $\rightarrow(3,0) \rightarrow(2,2) \rightarrow(4,1) \rightarrow(3,3) \rightarrow(2,1) \rightarrow(0,0) \rightarrow$ $(1,2) \rightarrow(-1,1) \rightarrow(0,3) \rightarrow(1,1)$ as shown in the third board arrangement in Figure 2. Sample boards are shown for the 2 


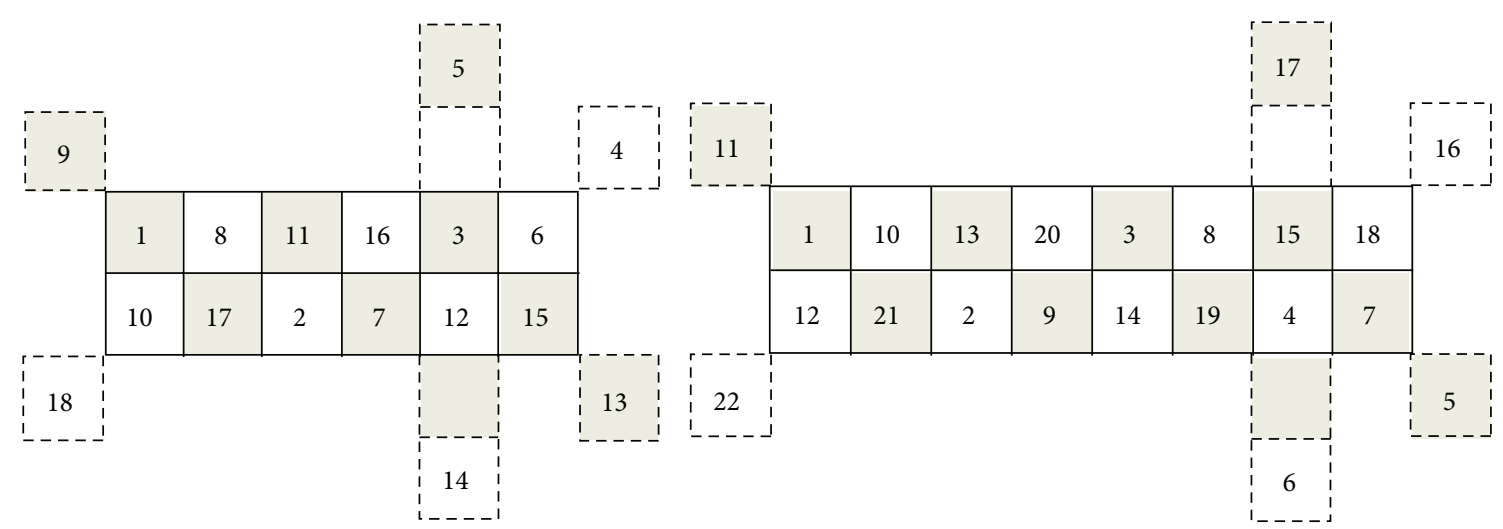

FIgURE 4 : The $2 \times 6$ and $2 \times 8$ boards illustrate that $\tau(2, n)=6$ if $n \geq 6$ is even.
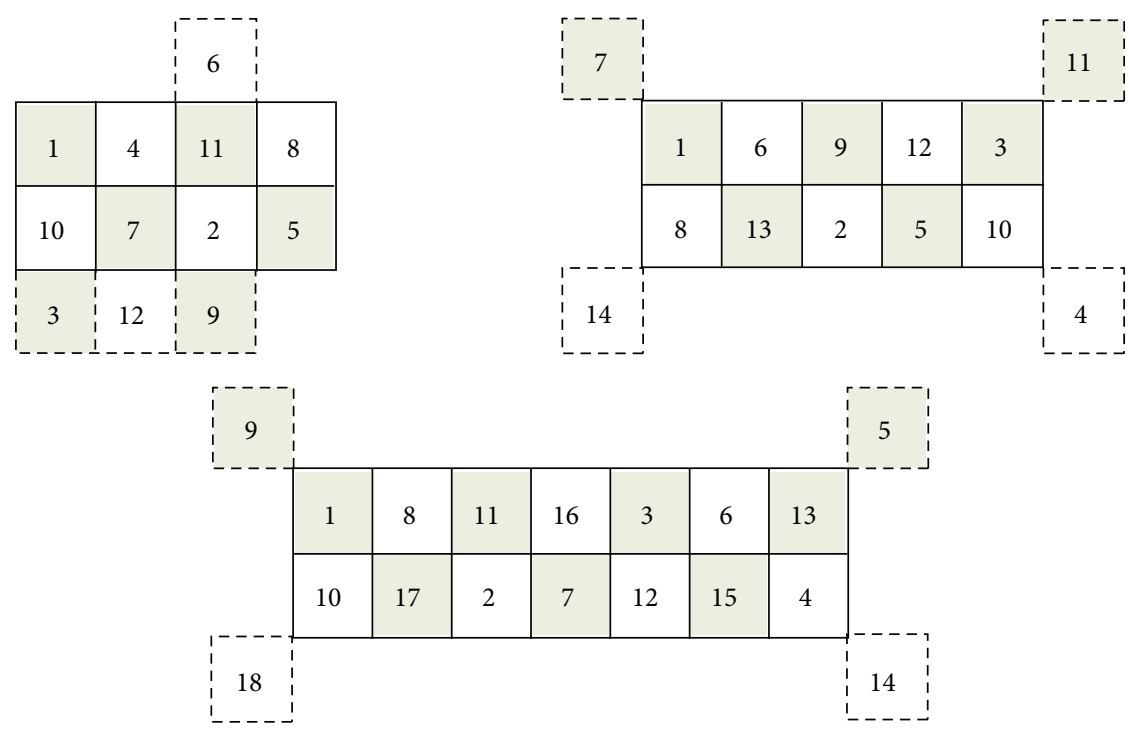

FIGURE 5: $\tau(2, n)=4$ if $n=4$ or is odd.
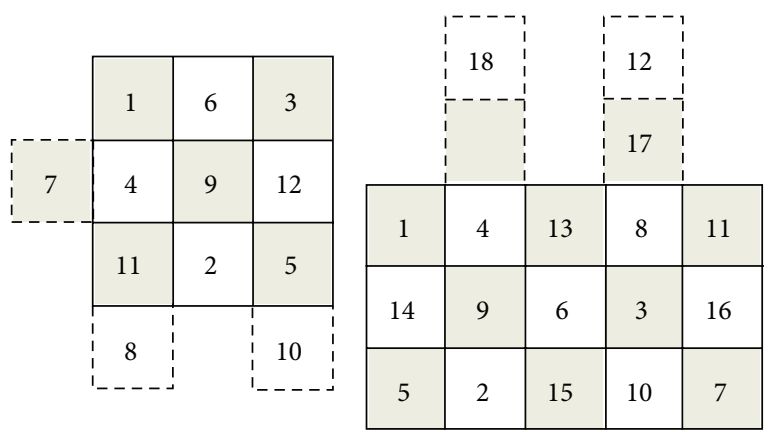

Figure 6: $\tau(3,3)=\tau(3,5)=3$.

$\times 6$ and $2 \times 8$ rectangles in Figure 4. Thus, $\tau(2, n)=6$ if $n \neq 4$ and is even.

If $n=4$, a knight at any square on the $2 \times 4$ board can move to only one other square, so the corresponding graph consists of four components (all paths with one edge). To form an extended closed knight's tour, at least four vertices must be added to connect the paths. This can be accomplished as shown in Figure 5.

Now consider when $n$ is odd. Again, the corresponding graph consists of four paths and at least four vertices are needed to connect them. This can be done by using one additional vertex (or square) at each corner of the chessboard and applying the following procedure.

(1) If $n=1+4 k$ for some positive integer $k$, then the paths are from $(1,1)$ to $(1, n),(2,1)$ to $(2, n),(1,2)$ to $(2, n-1)$, and $(2,2)$ to $(1, n-1)$.

If $n=3+4 k$ for some nonnegative integer $k$, then the paths are from $(1,1)$ to $(2, n),(2,1)$ to $(1, n),(1,2)$ to $(1$, $n-1)$, and $(2,2)$ to $(2, n-1)$.

(2) Insert the vertex $(3, n+1)$ between $(1, n)$ and $(2, n-1)$. Insert $(0,0)$ between $(1,2)$ and $(2,1)$. Insert $(0, n+$ $1)$ between $(2, n)$ and $(1, n-1)$. Finally, insert $(3,0)$ between $(2,2)$ and $(1,1)$. 


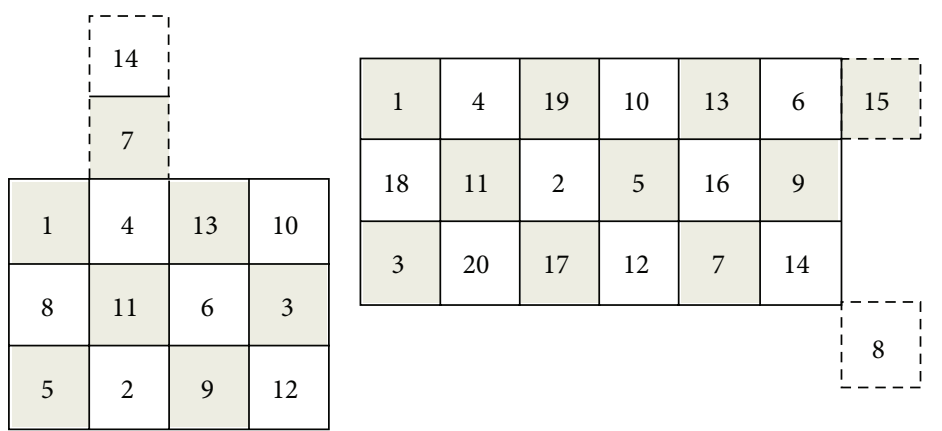

Figure 7: $\tau(3,4)=\tau(3,6)=2$.
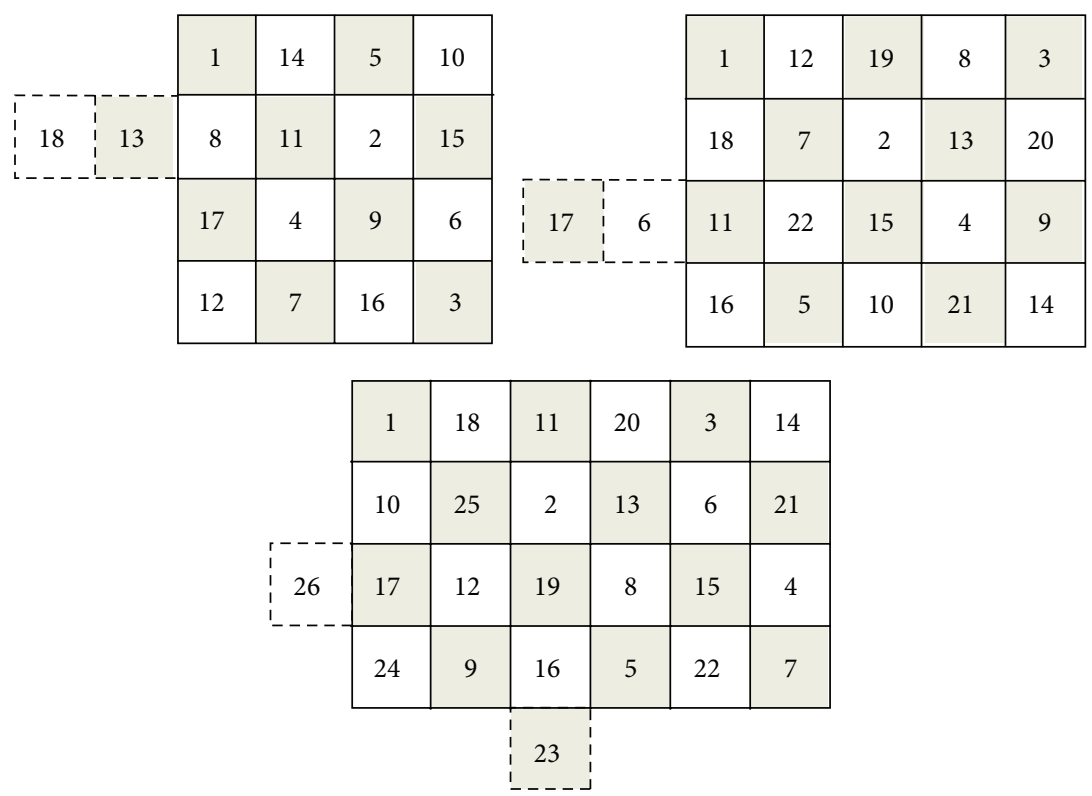

FIgURE 8: $\tau(4,4)=\tau(4,5)=\tau(4,6)=2$.

The extended closed knight's tours for the $2 \times 5$ and $2 \times 7$ boards are in Figure 5, and $\tau(2, n)=4$ if $n=4$ or is odd.

(c) The Case $m=3$. First, let $n=3$ or 5 . Since a $3 \times 3$ or $3 \times 5$ board has an odd number of squares, an odd number of squares must be added to complete an extended cycle (by observation (a)). For the $3 \times 3$ board, if the knight is on the center square, there are no squares to which it can move. Thus, an extra square is needed. Similarly, since the knight needs to return to the center square, a second square must be added. Therefore, $\tau(3,3) \geq 3$. In fact, exactly three additional vertices are needed as shown in Figure 6.

Suppose $\tau(3,5)=1$. Since square $(1,1)$ is black (per our assumptions), then the added square must be white (for parity) and must be adjacent to two black squares in the extended knight's tour. If we consider the cyclic graph associated with the tour and remove the added vertex, then a Hamiltonian path remains on the fifteen vertices with both endpoints corresponding to black squares. Since squares $(2,1)$ and $(2,5)$ are only adjacent to $(1,3)$ and $(3,3)$, the Hamiltonian path includes the cycle $(2,1),(1,3),(2,5),(3,3)$, and $(2,1)$, which cannot happen on a path. Therefore, $\tau(3,5)>2$ and is in fact 3, as shown in Figure 6.

Now, let $n=4,6$, or 8 . Based on observation (a), the extended tour numbers for the $3 \times 4,3 \times 6$, and $3 \times 8$ boards must be at least 2 . In fact, $\tau(3,4)=\tau(3,6)=2$ as shown in Figure 7 . To show that $\tau(3,8)=2$, we introduce a useful construction for larger boards that consists of combining a smaller board with a rectangle having an open knight's tour. For instance, to obtain a $3 \times 8$ board, attach the $3 \times 4$ board from Figure 1 to the right side of the $3 \times 4$ board in Figure 7 . Begin the closed knight's tour from square 1 to square 12, jump from square 12 to square $a$, follow the open knight's tour from $a$ to $l$, jump back from square $l$ to square 13, and resume the original closed knight's tour to square 1.

Finally, the proof that $\tau(3, n)=1$ if $n \geq 7$ and is odd is included in subcases 3 and 4 in the proof for case (e) below.

(d) The Case $m=4$. Since a $4 \times n$ board has an even number of squares, an even number of squares must be added to complete an extended cycle (by observation (a)). The $4 \times$ $4,4 \times 5$, and $4 \times 6$ boards are shown in Figure 8 . Each of 


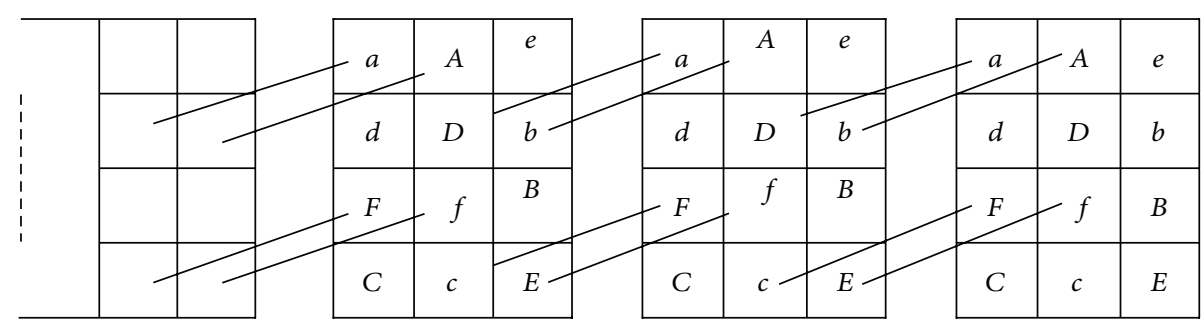

FIGURE 9: Connections between $4 \times 3$ expansion boards for $4 \times(n+3 k)$ boards with $n=4$, 5 , or 6 .

\begin{tabular}{|l|l|l|l|l|l|l|}
\multicolumn{9}{c|}{} & \\
\hline 1 & 20 & 17 & 14 & 11 & 8 & 5 \\
\hline 18 & 13 & 22 & 3 & 6 & 15 & 10 \\
\hline 21 & 2 & 19 & 12 & 9 & 4 & 7 \\
\hline
\end{tabular}

\begin{tabular}{|c|c|c|c|c|c|c|c|c|}
\hline \multicolumn{10}{c|}{} & \multicolumn{1}{l|}{1} \\
\hline 1 & 26 & 3 & 20 & 13 & 22 & 9 & 16 & 11 \\
\hline 4 & 19 & 28 & 25 & 6 & 17 & 14 & 23 & 8 \\
\hline 27 & 2 & 5 & 18 & 21 & 24 & 7 & 10 & 15 \\
\hline
\end{tabular}

\begin{tabular}{|c|c|c|c|c|}
\multicolumn{2}{|c|}{} & 26 & \multicolumn{2}{l|}{} \\
\hline 1 & 18 & 7 & 12 & 25 \\
\hline 8 & 13 & 24 & 17 & 22 \\
\hline 19 & 2 & 21 & 6 & 11 \\
\hline 14 & 9 & 4 & 23 & 16 \\
\hline 3 & 20 & 15 & 10 & 5 \\
\hline
\end{tabular}

\begin{tabular}{|c|c|c|c|c|c|c|}
\multicolumn{2}{l|}{} \\
\hline 1 & 32 & 17 & 8 & 25 & 30 & 15 \\
\hline 18 & 23 & 36 & 31 & 16 & 7 & 26 \\
\hline 33 & 2 & 9 & 22 & 27 & 14 & 29 \\
\hline 10 & 19 & 4 & 35 & 12 & 21 & 6 \\
\hline 3 & 34 & 11 & 20 & 5 & 28 & 13 \\
\hline
\end{tabular}

FIGURE 10: The $3 \times 7,3 \times 9,5 \times 5$, and $5 \times 7$ boards have extended tour numbers of 1 .

these "base" $4 \times n$ boards can be extended to a $4 \times(n+3 k)$ board by adding $k$ copies of a $4 \times 3$ board on the right and linking them as shown in Figure 9. For instance, if $k=1$, then the extended closed knight's tour is formed by altering two moves. Consider the $4 \times 4$ board and begin traversing at square 1 until you get to square 2 . Now replace the move from 2 to 3 with the following: from square 2, move to $a$, continue to $f$ (using the lowercase letters), and then return to square 3. Second, replace the move between squares 15 and 16 with the following: from square 15 , move to $A$, continue to $F$ (using the uppercase letters), and then return to square 16. Finally, complete the tour by continuing to 1 in the original $4 \times 4$ board. Similarly, for the $4 \times 5$ board use the tour $1 \rightarrow 2 \rightarrow$ $\cdots \rightarrow 13 \rightarrow a \rightarrow b \rightarrow \cdots \rightarrow f \rightarrow 14 \rightarrow 15 \rightarrow \cdots \rightarrow$ $20 \rightarrow A \rightarrow B \rightarrow \cdots \rightarrow F \rightarrow 21 \rightarrow 22 \rightarrow 1$, and for the $4 \times 6$ board use the tour $1 \rightarrow 2 \rightarrow \cdots \rightarrow 6 \rightarrow a \rightarrow b \rightarrow$ $\cdots \rightarrow f \rightarrow 7 \rightarrow 8 \rightarrow \cdots \rightarrow 21 \rightarrow A \rightarrow B \rightarrow \cdots \rightarrow$ $F \rightarrow 22 \rightarrow \cdots \rightarrow 26 \rightarrow 1$. If an extended closed knight's tour exists with $k-1$ copies of the $4 \times 3$ board, then the $k$ th copy can be attached by linking the squares $D, E, b$, and $c$ in the $(k-1)$ st copy with squares $a, f, A$, and $F$, respectively, in the $k$ th copy. Thus, $\tau(4, n)=2$ for $n \geq 4$. (e) The Case $m>4$ with $m$ and $n$ Both Being Odd. Figure 10 shows that,by observation (a), the values for $\tau(3,7)=$ $\tau(3,9)=\tau(5,5)=\tau(5,7)=1$. Finally, to show that $\tau(m, n)=1$ if $m$ and $n$ are odd with $3 \leq m, 7 \leq n$, we consider four subcases whose general structures are shown in (1). Each subcase is formed by constructing an $m \times n$ rectangle from the smaller rectangles in Figures 10, 1, and 11 and then "connecting" them according to the rules given in (2). In general, the tours begin in the upper left corner, move up and down the first column of boards, and travel across the rows of boards and back. The external square is always above the small rectangular board in the upper left corner of the $m \times n$ board. For clarity, note that, in subcase 1, if one begins the knight's tour with the upper left square labeled 1 , uses the labels for the squares of the boards as given in the figures, and follows the tour using the connecting rules, then the numbers and letters will always proceed in ascending order; however, in subcases 3 and 4 , when one uses rule 13 to connect the string of $3 \times 4$ boards in the top row, the second, fourth, sixth, and so forth copies of the board will be traversed in reverse order. That is, the labels will be selected from $l$ down to $a$ instead of from $a$ to $l$. Similarly, in subcases 2 and 4 , the 


\begin{tabular}{|c|c|c|c|}
\hline 12 & 17 & 8 & 3 \\
\hline 7 & 2 & 13 & 18 \\
\hline 16 & 11 & 4 & 9 \\
\hline 1 & 6 & 19 & 14 \\
\hline 20 & 15 & 10 & 5 \\
\hline
\end{tabular}

\begin{tabular}{|c|c|c|c|}
\hline$A$ & $e$ & $E$ & $c$ \\
\hline$H$ & $b$ & $B$ & $f$ \\
\hline$h$ & $D$ & $d$ & $F$ \\
\hline$a$ & $G$ & $g$ & $C$ \\
\hline
\end{tabular}

\begin{tabular}{|l|l|l|l|l|l|l|l|l|}
\hline 36 & 1 & 32 & 15 & 20 & 3 & 24 & 7 & 26 \\
\hline 31 & 14 & 19 & 2 & 33 & 10 & 27 & 4 & 23 \\
\hline 18 & 35 & 12 & 29 & 16 & 21 & 6 & 25 & 8 \\
\hline 13 & 30 & 17 & 34 & 11 & 28 & 9 & 22 & 5 \\
\hline
\end{tabular}

\begin{tabular}{|c|c|c|c|c|c|c|}
\hline 3 & 28 & 1 & 18 & 9 & 22 & 7 \\
\hline 14 & 17 & 4 & 25 & 6 & 19 & 10 \\
\hline 27 & 2 & 15 & 12 & 21 & 8 & 23 \\
\hline 16 & 13 & 26 & 5 & 24 & 11 & 20 \\
\hline
\end{tabular}

Figure 11: The additional $5 \times 4,4 \times 4,4 \times 7$, and $4 \times 9$ boards needed for the four subcases.

entire third, fifth, seventh, and so forth row of boards will be traversed in reverse order.
The four subcases for the general case when $m$ and $n$ are both odd are as follows:

Subcase 1. $m=1 \bmod 4, n=1 \bmod 4$

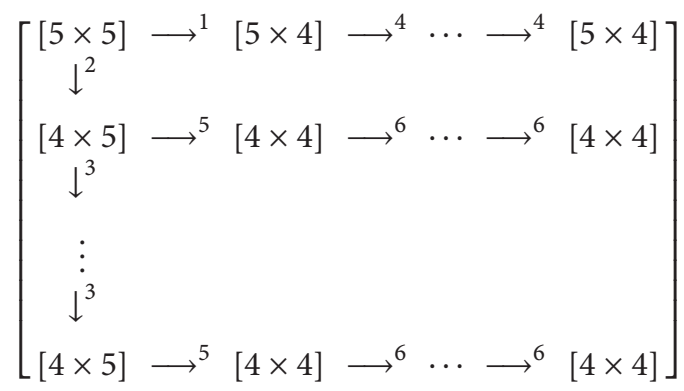

Subcase 2. $m=1 \bmod 4, n=3 \bmod 4$

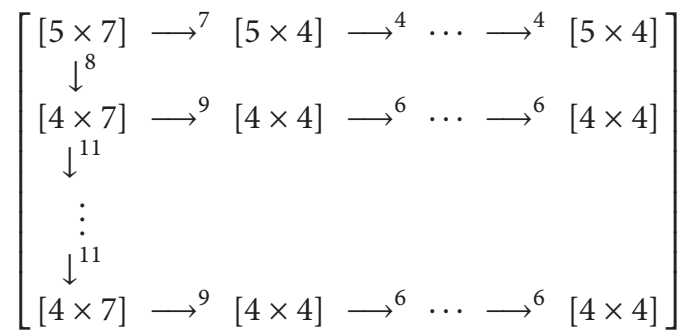


Subcase 3. $m=3 \bmod 4, n=1 \bmod 4$

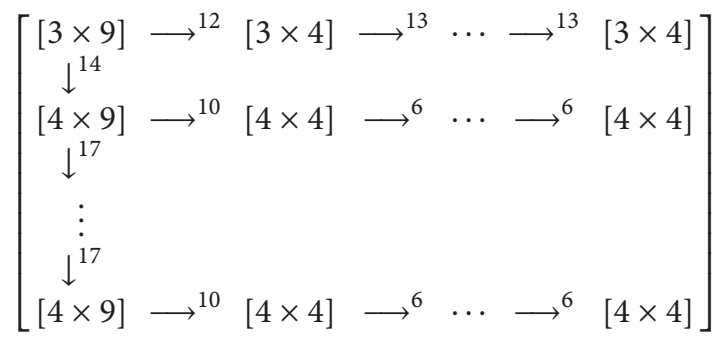

Subcase 4. $m=3 \bmod 4, n=3 \bmod 4$

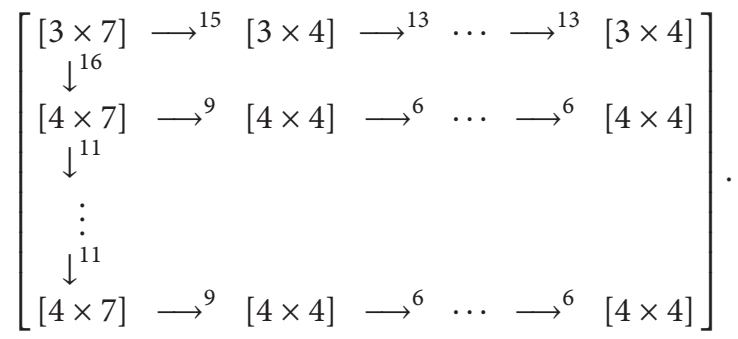

Connection rules for the four subcases are as follows:

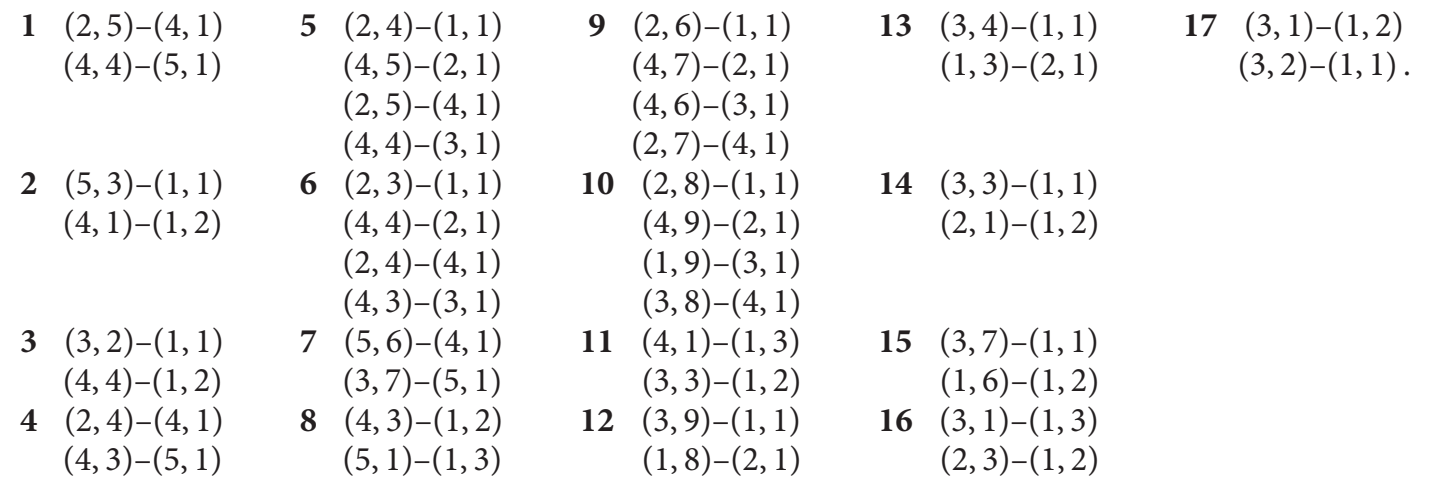

\section{References}

Every rectangular board has an extended knight's tour and the extended tour numbers are summarized in Theorem 1, and the constructions in the proof indicate where the extended squares should be.

\section{Conflict of Interests}

The authors declare that there is no conflict of interests regarding the publication of this paper.

\section{Acknowledgment}

The authors wish to thank the referees and the editor for helpful suggestions which improved the mathematical accuracy and the presentation of this paper.
[1] J. J. Watkins, Across the Board: The Mathematics of Chessboard Problems, Princeton University Press, Princeton, NJ, USA, 2004.

[2] A. J. Schwenk, "Which rectangular chessboards have a knight's tour?” Mathematics Magazine, vol. 64, no. 5, pp. 325-332, 1991.

[3] J. Demaio and T. Hippchen, "Closed knight's tours with minimal square removal for all rectangular boards," Mathematics Magazine, vol. 82, pp. 219-225, 2009.

[4] D. E. Knuth, "Leaper graphs," The Mathematical Gazette, vol. 78, pp. 274-297, 1994.

[5] A. M. Miller and D. L. Farnsworth, "Knight's tours on cylindrical and toroidal boards with one square removed," Ars Combinatoria, vol. 108, pp. 327-340, 2013. 
[6] J. Erde, B. Golénia, and S. Golénia, "The closed knight tour problem in higher dimensions," The Electronic Journal of Combinatorics, vol. 19, no. 4, pp. 9-17, 2012.

[7] J. DeMaio and B. Mathew, "Which chessboards have a closed knight's tour within the rectangular prism?" Electronic Journal of Combinatorics, vol. 18, no. 1, pp. 8-14, 2011.

[8] N. Kamčev, “Generalised Knight's tours," Electronic Journal of Combinatorics, vol. 21, no. 1, pp. 1-31, 2014.

[9] S. Bai, X.-F. Yang, G.-B. Zhu, D.-L. Jiang, and J. Huang, "Generalized knight's tour on 3D chessboards," Discrete Applied Mathematics, vol. 158, no. 16, pp. 1727-1731, 2010.

[10] G. L. Chia and S.-H. Ong, "Generalized knight's tours on rectangular chessboards," Discrete Applied Mathematics, vol. 150, no. 1-3, pp. 80-98, 2005.

[11] G. Bullington, L. Eroh, S. J. Winters, and G. L. Johns, "Variations for the knights tour for rectangular chessboards on alternative surfaces," Congressus Numerantium, vol. 206, pp. 199-214, 2010. 


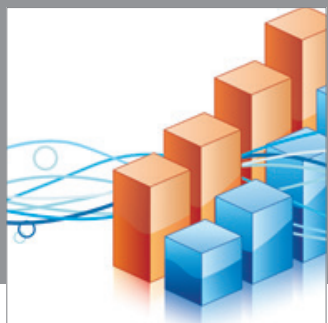

Advances in

Operations Research

mansans

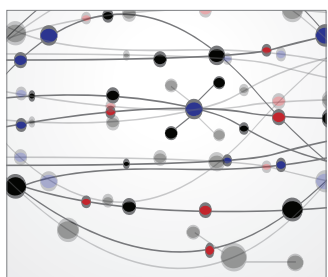

The Scientific World Journal
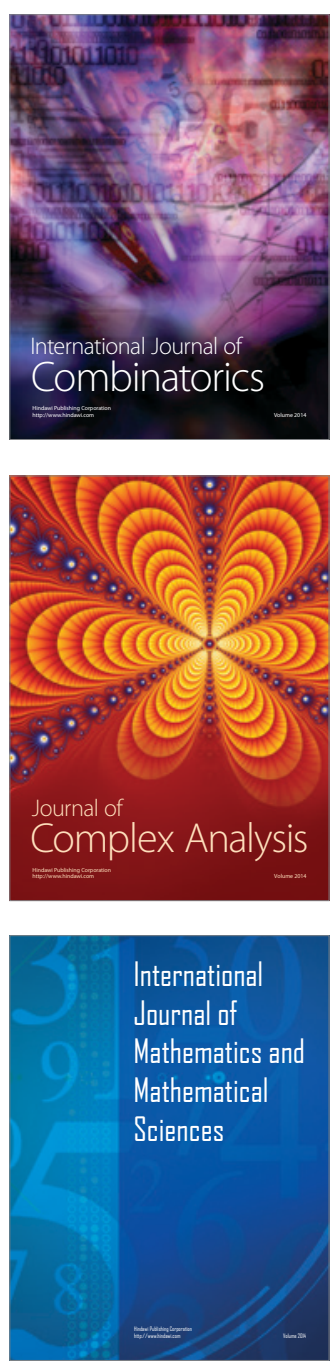
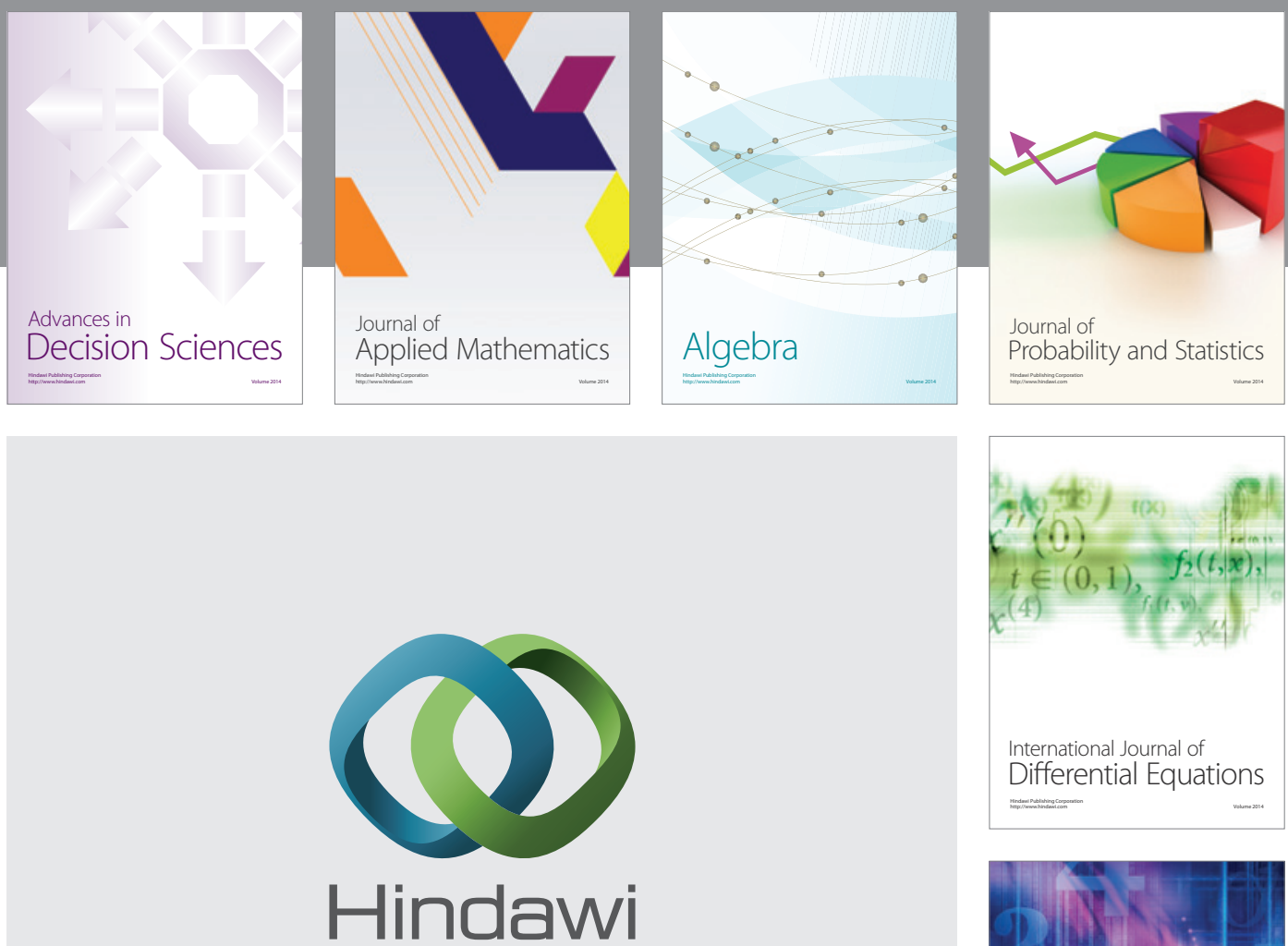

Submit your manuscripts at http://www.hindawi.com
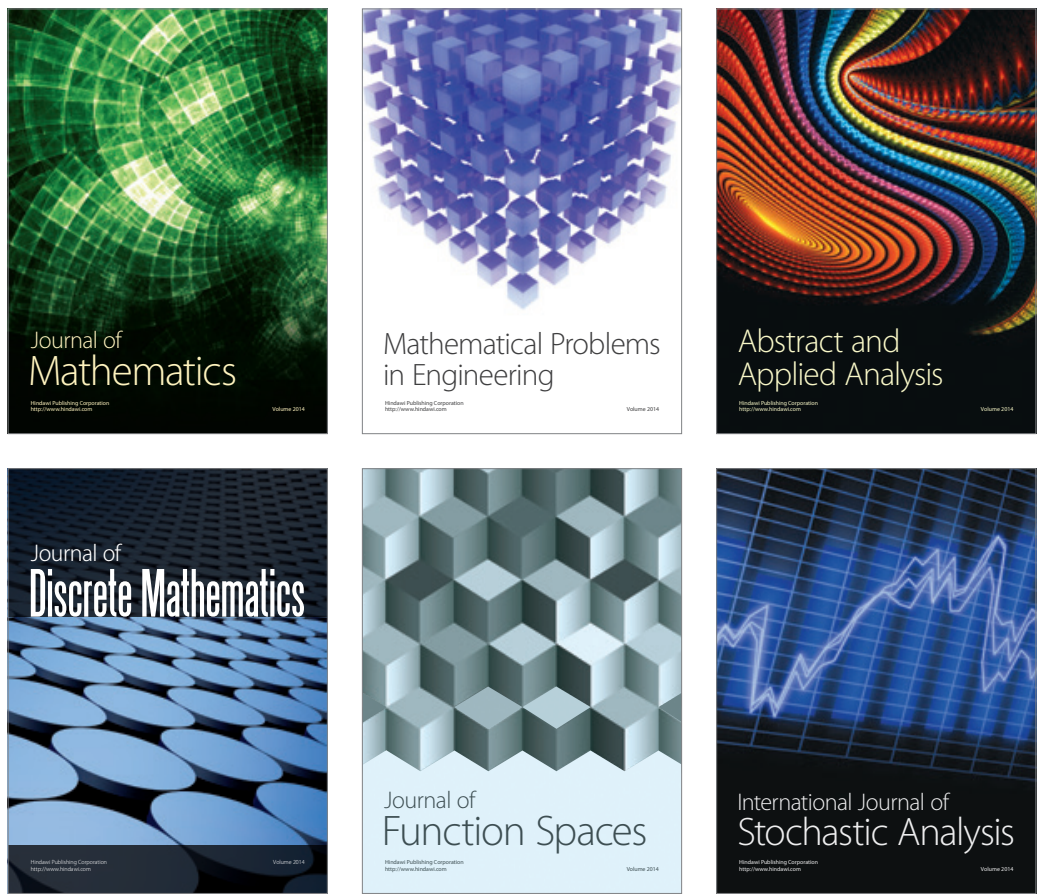

Journal of

Function Spaces

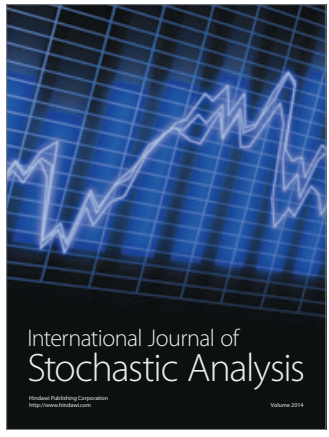

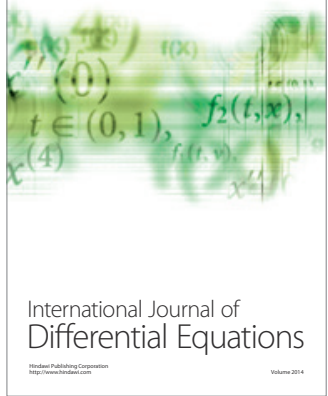
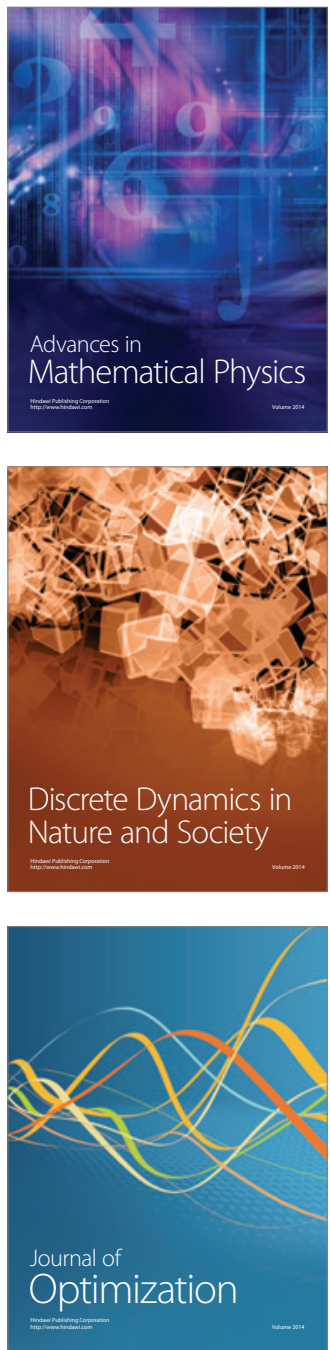